\title{
A Numerical Study of Initiation and Migration of Trapped Oil in Capillaries with Noncircular Cross Sections
}

\author{
Long Long $\mathbb{D}^{1,2}$ Yajun $\mathrm{Li}^{2}{ }^{2}$ Houjian Gong, ${ }^{2}$ and Mingzhe Dong ${ }^{3}$ \\ ${ }^{1}$ Institute of Mud Logging Technology and Engineering, Yangtze University, Jingzhou, Hubei 434000, China \\ ${ }^{2}$ School of Petroleum Engineering, China University of Petroleum, Qingdao, Shandong 266580, China \\ ${ }^{3}$ Department of Chemical and Petroleum Engineering, University of Calgary, Calgary, AB, Canada T2N 1N4
}

Correspondence should be addressed to Long Long; longlong736353@126.com

Received 6 May 2019; Accepted 28 July 2019; Published 8 August 2019

Academic Editor: Stefano Lo Russo

Copyright (c) 2019 Long Long et al. This is an open access article distributed under the Creative Commons Attribution License, which permits unrestricted use, distribution, and reproduction in any medium, provided the original work is properly cited.

To clarify the initiation and migration mechanisms of a discontinuous oil phase in pores, a numerical study was performed to interpret the starting phenomenon and flowing rules of oil trapped in capillaries that have noncircular cross sections. In this study, capillaries with three different cross sections were used to investigate the deformation law of oil and the pressure drop across these microchannels at different displacement velocities by computational fluid dynamics (CFD). The geometrical structure of the microchannels was precisely controlled, and the migration process of the oil, which is too small to be observed by direct experimentation, was assessed and quantitatively analyzed. By analyzing the shape of the trapped oil after reaching a steady state at different velocities, the nonstart and start conditions could be distinguished and the accuracy of the numerical method was verified by a comparison with an analytical method (the MS-P method). Two aspects of oil migration in noncircular microchannels were observed in combination with previous studies: there is a driving force on the cross section of the oil drop and a viscous force at the oil-water interface in the corners, and the more irregular the pore section is, the more easily the trapped oil will migrate. Additionally, the influence of the microchannel cross-sectional shape on the non-Darcy flow of a discontinuous oil phase was clarified. It can be concluded that the presence of the non-Darcy flow in pores arises because trapped oil, as a discontinuous phase, cannot be separated from the capillary wall without reaching critical velocity.

\section{Introduction}

Seventy percent of global crude oil is produced from the mature oilfields, which have entered a high water-cut development stage and have a recovery rate of approximately only $30 \%$. Approximately $2 / 3$ of residual oil remains dispersed in underground reservoirs, which has great potential for recovery. After long-term water injection development, the residual oil in the porous media of the reservoir is gradually transformed from a continuous phase to a discontinuous phase $[1,2]$. A large amount of residual oil is in the form of a single-hole column or a porous column and is retained in the pore and throat of the porous media in the form of trapped oil with different lengths, shown as Figure 1. When the crude oil in the porous media is in a discontinuous phase, the oil's mechanism of migration is different from that in a continuous phase. Experimental results have shown that there is a significant starting phenomenon (initiation) in the early stages of discontinuous oil phase migration in microchannels $[3,4]$. During the initiation process, the discontinuous oil phase has three deformation stages: first, it contacts the inner wall completely, then it contacts the inner wall partially, and finally, it completely separates from the wall, and the pressure drop (or resistance coefficient) in the discontinuous oil phase is much larger than the value calculated by Darcy's law. The pressure drop indicates that very high additional resistance 


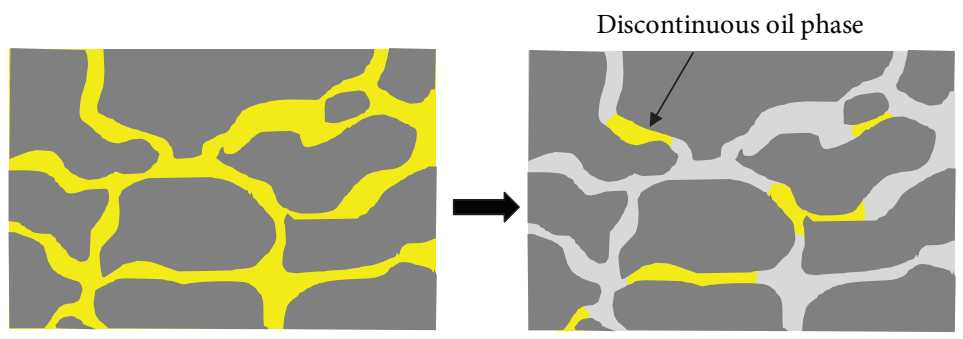

(a)

(b)

Figure 1: The residual oil in the porous media of the reservoir: (a) before water flooding; (b) after water injection development.

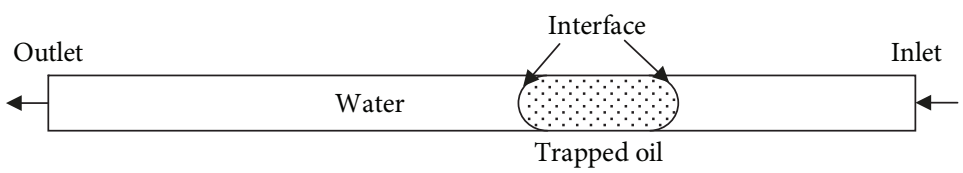

(a)

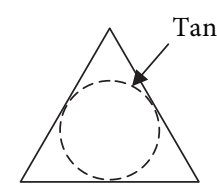

(b)

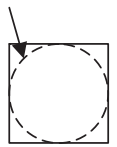

(c)

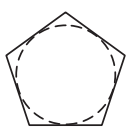

(d)

Figure 2: Modeling of the flow in capillaries: (a) side view of oil trapped in a capillary, (b) equilateral triangle cross section, (c) quadrilateral cross section, and (d) pentagon cross section.

will occur in the pore during the start process, which will hinder the migration of the discontinuous oil phase in the porous media. This phenomenon complicates the oil-water two-phase seepage mechanism, and it is difficult to accurately describe the flow law of the discontinuous phase fluid in the porous media using the traditional reservoir engineering method.

To clarify the starting mechanism of the discontinuous oil phase, scholars have used the circular cross-sectional microchannel model to carry out experimental and theoretical studies on the discontinuous phase [5-12]. The flow of the discontinuous phase in a circular cross-sectional microchannel can be qualitatively analyzed, and many meaningful conclusions and understandings can be obtained. Based on a bubble flow experiment in a circular section capillary, Fairbrother and Stubbs [13] found that when the discontinuous phase migrates in a capsule shape in the microchannel, a water film is formed between the inner wall and the discontinuous phase and the moving velocity of the bubble is greater than the average flow velocity of the liquid. Other researchers have carried out a large number of experimental studies on the discontinuous phase flow in circular microchannels and have obtained calculation formulas for the discontinuous phase velocity in different capillary numbers [14-17]. However, the capillary number, Ca, considered in these studies was $10^{-3}-10^{-2}$, which is much higher than the $\mathrm{Ca}$ value in the pores of porous media [18-20]. Therefore, the discontinuous phase starting phenomenon has not been fully clarified by previous research.

Dong et al. [3] discovered the "starting" phenomenon of retained oil droplets through a trapped oil droplet flooding experiment in a circular cross-sectional microchannel with a capillary number of $10^{-7}-10^{-6}$. By measuring the pressure drop across the microchannel during oil drop deformation with a high sensitivity pressure sensor, the authors proposed three stages of the discontinuous oil phase starting process: the build-up stage, the hold-up stage, and the steady flow phase. The discontinuous oil phase is in full contact with the inner wall during the build-up stage. Upon an increase in the injection volume, the water film gradually spreads and wraps the discontinuous oil in the hold-up stage. After being completely surrounded by the water film, the discontinuous oil phase separates from the inner wall and enters the steady flow stage. The experimental results show that the discontinuous oil phase is in contact with the inner wall before starting (hold-up stage and steady flow phase) and the pressure drop (or resistance coefficient) across the microchannel is much higher than the pressure drop calculated by Darcy's law in that stage.

For this phenomenon, Dai et al. [21-23] simulated the migration of oil droplets in circular cross-sectional microchannels by experimental and computational fluid dynamics methods and found that the formation of a water film between the oil droplets and the inner wall has a large impact on the flow of oil droplets. Hsu and Hilpert [24] found that in the presence of a water film, the classical Young-Laplace equation is not sufficient to describe the droplet starting process and the corresponding pressure drop changes. To clarify the influence mechanism of the water film, Long et al. [4] proposed an equivalent viscosity model to explain the formation of additional resistance in the microchannel during the discontinuous oil phase 


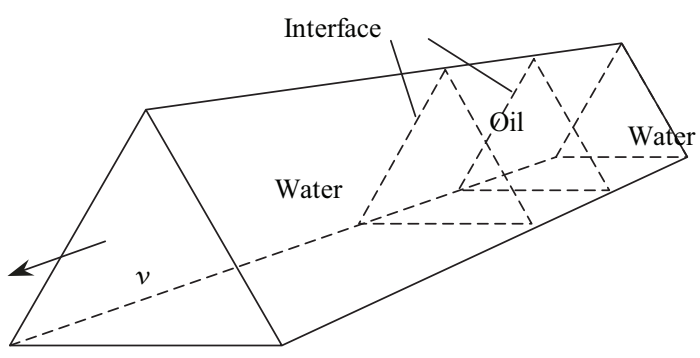

(a)

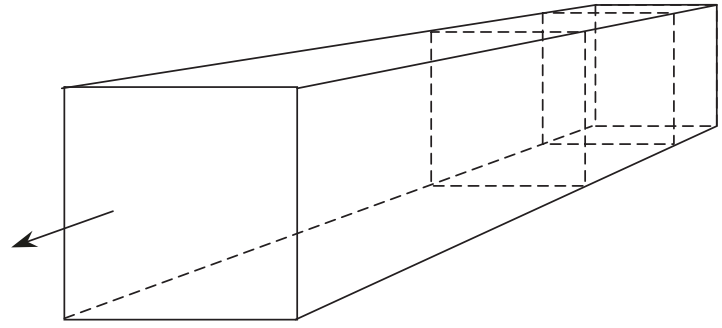

(b)

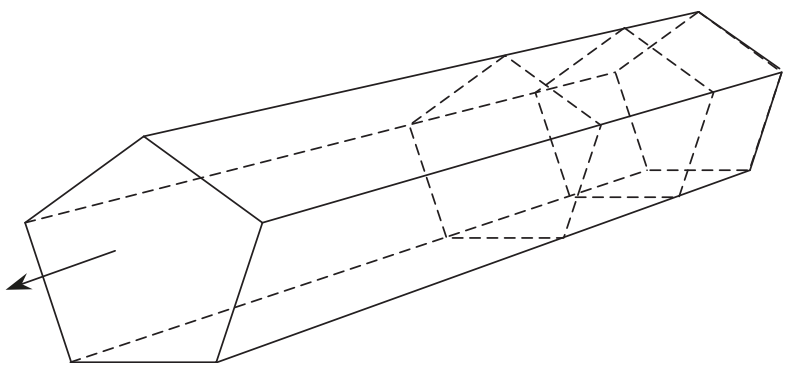

(c)

FIGURE 3: Initialization state diagram: (a) equilateral triangular cross section, (b) quadrilateral cross section, and (c) pentagonal cross section.

TABLE 1: Fluid properties of oil and water.

\begin{tabular}{lcc}
\hline Fluid & Density $\left(\mathrm{kg} \cdot \mathrm{m}^{-3}\right)$ & Viscosity $(\mathrm{Pa} \cdot \mathrm{s})$ \\
\hline Water & 998 & 0.001 \\
Oil & 780 & 0.1 \\
\hline
\end{tabular}

starting phenomenon based on morphological changes and pressure drop changes.

These studies have further deepened the understanding of the phenomenon of discontinuous oil phase initiation, but they have not revealed the mechanism of discontinuous oil phase initiation and migration in the pores. Due to the geometrical complexity of pores in real porous media, the water phase occupied the corners of the cross section, affecting the initiation and migration of the discontinuous oil phase. However, there are few reports on the effect of water channels in the corners on the start and migration of the discontinuous oil phase [25-29]. Therefore, the study of discontinuous oil phase migration in microchannels with a noncircular cross section may help to explain the nonDarcy phenomenon of porous media seepage from a microscopic point of view.

In this research, we aim to reveal the critical conditions for discontinuous oil phase initiation in pores and the influence of the pore cross section on the non-Darcy flow of the discontinuous oil phase. By using computational fluid dynamics (CFD) methods, numerical simulation is carried out to study the initiation and migration of the trapped oil in capillaries with triangular, quadrilateral, and pentagonal cross sections. The formation and deformation of the oil drops are discussed in detail. Compared with the analytical solutions obtain by the MS-P method [30, 31], the accuracy of the simulations are also verified. Meanwhile, by comparing the Darcy flow results in a circular tube, the influence of the cross section on the non-Darcy flow of the discontinuous oil phase in microchannels is discussed.

\section{Establishment and Algorithm of the CFD Model}

2.1. Governing Equations. The CFD software ANSYS 14.5 is used to simulate the initiation and migration of the trapped oil noncircular cross-sectional capillaries, while the immiscible fluid (water and oil) interface is traced by the volume of fluid (VOF) method. For a Newtonian and incompressible fluid, the continuity equation is given as

$$
\frac{\partial \rho}{\partial t}+\nabla \cdot(\rho u)=0
$$

In addition, the Navier-Stokes equation, as the momentum equation, can be written as

$$
\frac{\partial}{\partial t}(\rho u)+u \cdot \nabla u=\nabla p+\mu \nabla^{2} u+f
$$

Here, $u$ is the flow velocity, $p$ is pressure, and $f$ is the body force per unit volume. The values of $\rho$ and $\mu$ denote the average density and viscosity, respectively.

2.2. Modeling of Flow in Capillaries. To understand the influence of the pore cross section on the non-Darcy flow, capillaries with different cross sections are used to investigate the initiation and migration of discontinuous oil. Three kinds of cross sections are used in this study, namely, equilateral triangle, quadrilateral, and pentagon, with the same radius as the tangential circle. The configurations of the different capillary cross sections are shown in Figure 2. In the geometry model, the length of the microchannel is $10 \mathrm{~mm}$, with a tangential circle radius of 

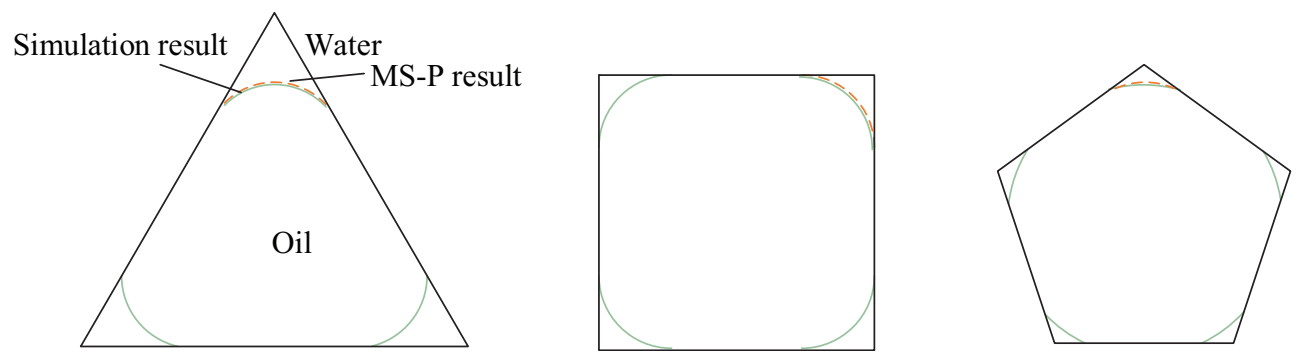

FIGURE 4: Interfaces obtained by the simulation and by the MS-P method.

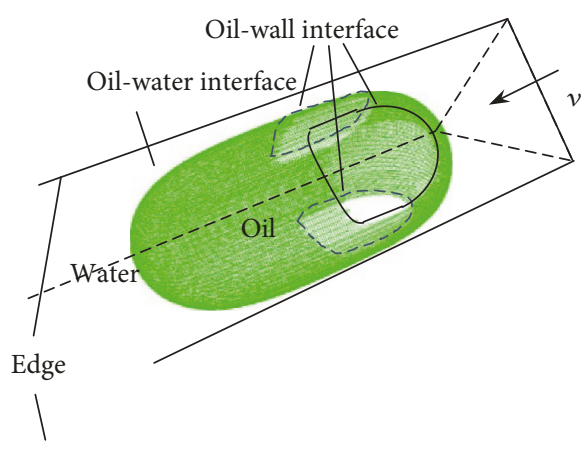

(a)

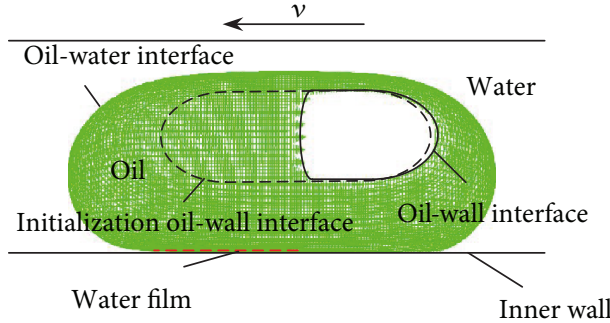

(c)

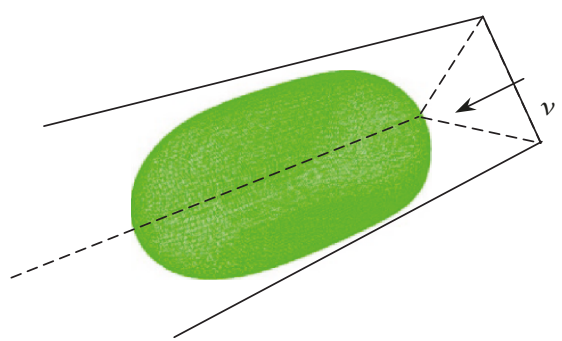

(b)

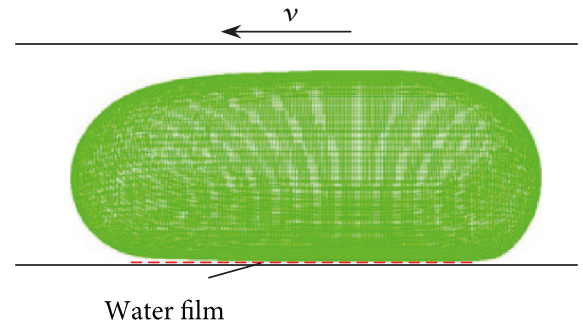

(d)

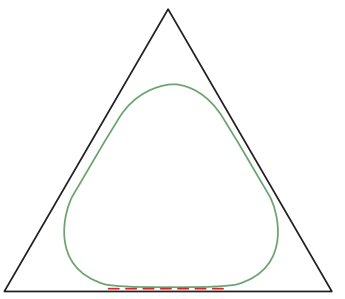

(f)

FIGURE 5: The oil-water interfaces in an equilateral triangle cross-sectional capillary: (a) three-dimensional view of the nonstart condition, (b) three-dimensional view of the start condition, (c) side view of the nonstart condition, (d) side view of the start condition, (e) cross-sectional view of the nonstart condition, and (f) cross-sectional view of the start condition.

$0.5 \mathrm{~mm}$. In terms of boundary conditions, the velocity inlet and pressure outlet are used in this study and the inner wall is a nonslip boundary. To improve the resolution of the simulation, boundary layer tools are used in the inner wall of the three cases: there are 10 layers near the wall, and the width of the first layer is $10 \mu \mathrm{m}$, with a growth factor of 1.1. Quadrilateral meshes are used in the cross sections of the three models. Because of the different cross-sectional shapes, the three cross sections have differ- ent numbers of meshes. The total grid numbers of the equilateral triangle, quadrilateral, and pentagon cross sections are 950000,1250000 , and 1000000 , respectively.

In the initialization setting, oil with a $2 \mathrm{~mm}$ flat end is placed in the capillary, and the distance between the rear interface and the inlet is $3 \mathrm{~mm}$; the initialization states of the three cases are shown in Figure 3. Under the action of the capillary force, the front and rear interfaces transform into a meniscus [4]. By the UDF function, the inlet velocity 
increases from zero to $0.1 \mathrm{~m} / \mathrm{s}$ after the oil reached a steady condition [32]. During the flow simulation, the capillary number ranges from $2.5 \times 10^{-5}$ to $2.5 \times 10^{-3}$, and the Reynolds number is less than 99.8 , indicating a laminar flow. Meanwhile, the outlet pressure is set to zero. The density and viscosity of the oil and water are shown in Table 1, and the contact angle and interfacial tension between them are set as $0.5^{\circ}$ and $0.04 \mathrm{~N} / \mathrm{m}$, respectively.

There are several additional assumptions in this numerical study: (1) compared with the capillary force, the effect of gravity can be neglected; (2) as mentioned above, because of the low Reynolds number, the flow can be regarded as a laminar flow; and (3) the influence of the meniscus appearance and distance between the rear interface and the inlet on the length of oil is negligible.

\section{Results and Discussion}

3.1. Initialization, Start, and Migration of Trapped Oil. At the start of the simulation, the velocity of the inlet is set as zero by the UDF function and the trapped oil begins to deform under the action of capillary force. The flat interfaces of the front and rear become menisci, and water channels form at the corners. The oil maintains contact with the inner walls. Moreover, the interfaces of the cross sections are in agreement with the results of the MS-P method, as shown in Figure 4. In the figure, the green lines in the cross sections represent the oil-water interfaces that are obtained by CFD simulation, and the orange dotted lines are the menisci calculated by the MS-P method.

After the oil reaches the initialization state, the inlet velocity gradually increases and the oil begins to move along the displacement direction. The velocity used in this research ranges from $0.001 \mathrm{~m} / \mathrm{s}$ to $0.1 \mathrm{~m} / \mathrm{s}$, while the maximum Reynolds number is less than 99.8 .

In the migration stage, the oil moves forward, driven by water, and the rear and front menisci become slightly deformed. The rear interface gradually flattens and the curvature of the front meniscus increases. Meanwhile, water films appear between the oil and inner walls, which decrease the area of the oil-wall interface. When the velocity is fixed, the migration pattern of the oil reaches a steady state with the progress of displacement. However, the steady state of the oil varies under different velocities. At low velocity, the oil cannot be separated from the inner walls when it reaches a steady state, which can be identified as a nonstart condition. In the steady state of oil under higher velocities, the oil no longer remains in contact with the inner wall, which is identified as the start condition.

Figure 5 shows the nonstart and start conditions of the oil under different steady states in the equilateral triangle cross-sectional capillary. Figures 5(a), 5(c), and 5(e), respectively, depict a three-dimensional view, side view, and cross-sectional view of the oil-water interface under the nonstart condition $(0.01 \mathrm{~m} / \mathrm{s})$. The corresponding views of the start condition are shown in Figures 5(b), $5(\mathrm{~d})$, and $5(\mathrm{f})(0.1 \mathrm{~m} / \mathrm{s})$. In the nonstart condition in the equilateral triangle cross section (Figure 5(a)), the oil-water interface is displayed as a green capsular surface, with oil

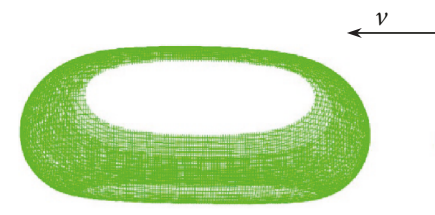

$0.002 \mathrm{~m} / \mathrm{s}$

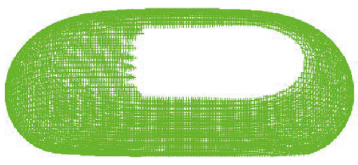

$0.008 \mathrm{~m} / \mathrm{s}$

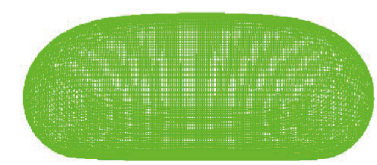

$0.015 \mathrm{~m} / \mathrm{s}$

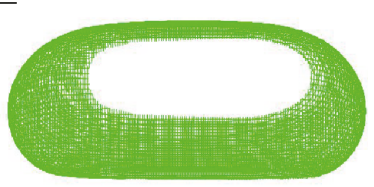

$0.005 \mathrm{~m} / \mathrm{s}$

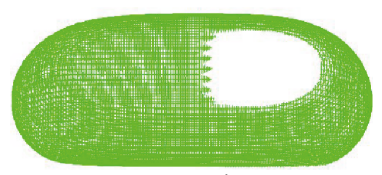

$0.01 \mathrm{~m} / \mathrm{s}$

$0.05 \mathrm{~m} / \mathrm{s}$

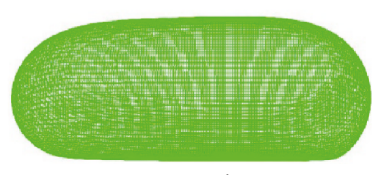

Figure 6: Side views of the oil at different velocities.

inside and water outside, and the bullet-like gaps on it represent the oil-wall interfaces on the three inner walls. When comparing the steady-state oil-wall interface with the initialization oil-wall interface, shown in Figure 5(c), it is seen that the contact area between the oil and inner walls is reduced due to the formation and spread of the water film, which is displayed as a red dotted line. Furthermore, on the cross section (Figure 5(e)), the oil-water interfaces moves from the initial position (orange dotted line) to the center of the tube. This change indicates that the water channels at the corners become larger. According to previous research [31], there is a lubricating effect of low-viscosity fluids on high-viscosity fluids, which are water and oil, respectively, in this study. Therefore, it is reasonable to believe that the water channels would increase with an increase in the oil flow capacity.

When the inlet velocity is large enough, the steady state of oil is the start condition. The oil separates from the walls, and the oil-water interface displays a perfectly capsular shape, with no gaps, as shown in Figure 5(b). Otherwise, it denotes that there is no oil-wall interface and the water film completely spreads and wraps the oil, as indicated by the red dotted line though the oil in Figure 5(d). In the crosssectional view, the area of the water channels are further expanded compared to the nonstart condition. This result also confirms the hypothesis regarding the influence of the water channels on oil flow capacity.

Figure 6 depicts the side views of the oil-water interface under steady-state conditions for different velocities. By comparing the interfaces at different velocities, it is found that when the speed is less than $0.01 \mathrm{~m} / \mathrm{s}$, the interface in the steady state is discontinuous and the oil remains in contact with the inner wall. Additionally, when the velocity increases, the contact area between the oil and inner wall decreases and the oil-wall interfaces are biased to the rear meniscus. This phenomenon illustrates that the water films spread between the oil and inner walls upon an increased velocity in the nonstart conditions. The cases of $0.015 \mathrm{~m} / \mathrm{s}$ and $0.05 \mathrm{~m} / \mathrm{s}$ are in a start condition, but the oil in a higher 


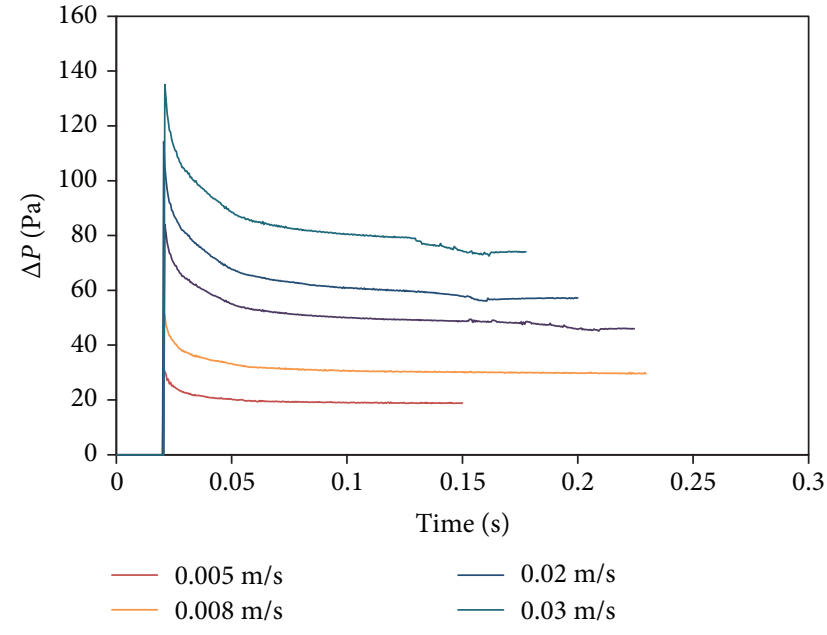

(a)

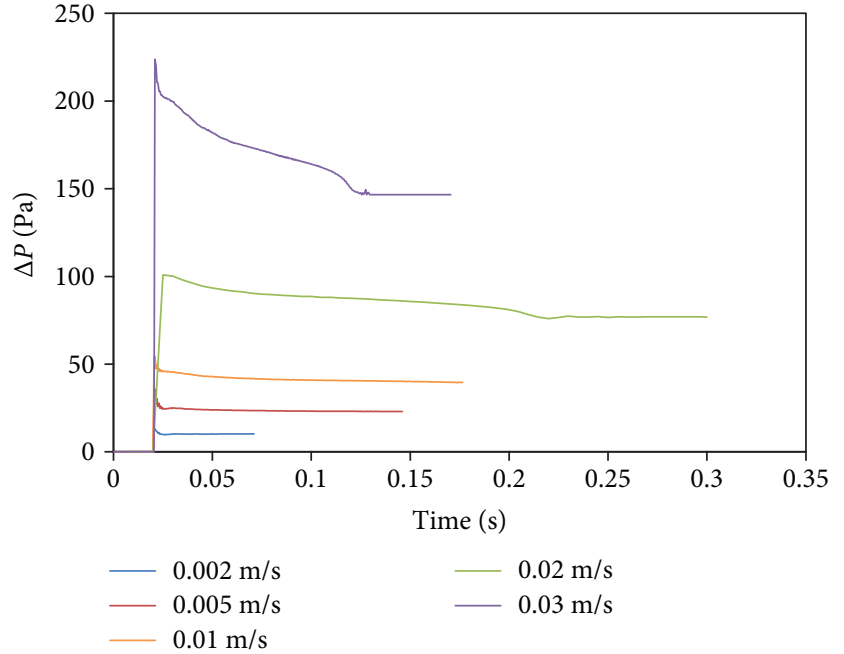

(b)

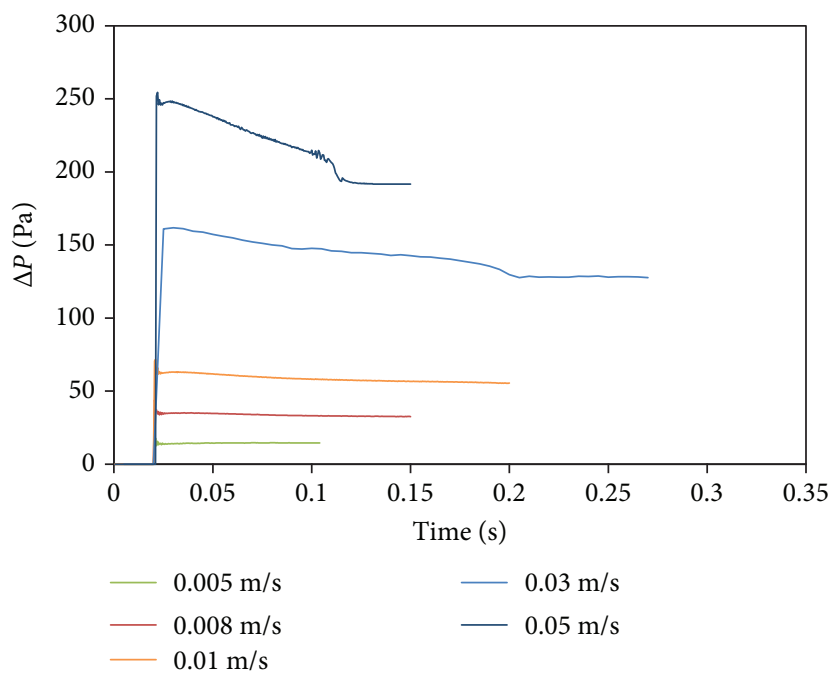

(c)

Figure 7: Pressure drops curves for different velocities in capillaries: (a) triangle cross section, (b) quadrilateral cross section, and (c) pentagon cross section.

velocity condition becomes more slender, which means the water channels in the corners become larger.

In the capillaries with equilateral, quadrilateral, and pentagon cross sections, the trapped oil has similar migration characteristics, which are depicted in Figures 5 and 6. Due to the different sizes of water channels caused by the different geometrical shapes, the critical velocity of oil reaching a start condition varies. These influences are discussed in the next section.

3.2. Pressure Drop in Different Capillaries. To analyze the characteristics of a two-phase discontinuous flow in capillaries with different cross-sectional shapes, the pressure drop curves of the capillaries were studied and compared. Figure 7 shows the pressure drop curves for different velocities in capillaries with equilateral triangle, quadrilateral, and pentagon cross sections. In the equilateral triangle cross-sectional capillaries, shown in Figure 7(a), the pressure drop across the microchannel is zero at initialization, i.e., at 0.02 seconds. As the oil moves, the pressure drop rises and then gradually returns to a stable value, and the greater the velocity is, the greater the pressure drop is. When the displacement velocity is small (i.e., $0.005 \mathrm{~m} / \mathrm{s}$ and $0.008 \mathrm{~m} / \mathrm{s}$ ), the pressure drop changes directly. In these cases, as the pressure drop returns to a stable value, a steady state is reached. At these velocities, the oil is not started and remains in contact with the walls. However, when the velocity is large enough (more than $0.015 \mathrm{~m} / \mathrm{s}$ ), the pressure drop varies in two stages. In the first stage, the pressure drop continues to decrease and the downward trend is nearly linear; this trend is more obvious in Figures 7(b) and 7(c). The oil maintains contact with the inner walls at this stage, but the contact area is gradually reduced. After the first stage, the pressure drop decreases steeply and enters the second stage, eventually stabilizing. This trend indicates that the oil has been started and leaves the wall in a steep descent. When the oil becomes detached, 


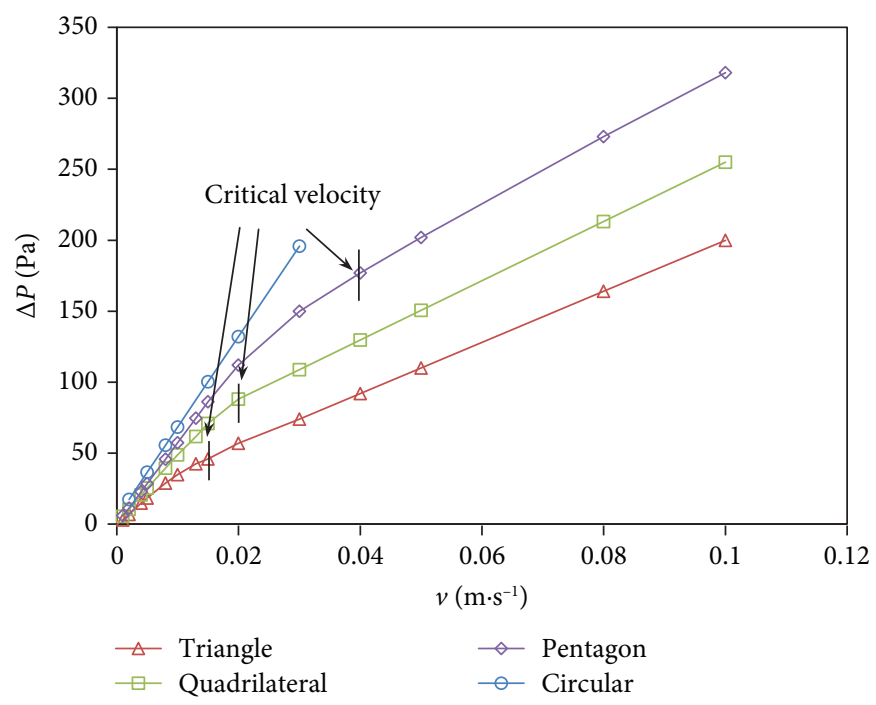

Figure 8: Steady pressure drop vs. velocity in different capillaries.

the pressure drop immediately reaches a stable value. The phenomenon of the pressure drop varying in stages has also been shown in experimental and numerical studies on trapped oil in capillaries with circular cross sections $[3,4]$.

As seen in the three graphs in Figure 7, the velocities at which the trapped oils separate from the walls of the different cross sections are different. The oil in the triangular crosssectional capillary is the first to be separated, the square cross section is the second, and the regular pentagonal cross section is the last. According to previous research [33] and the results of the current study (Figure 4), when the number of sides of a cross section increases, and the high-order polygon gradually approaches a circle, the saturation of the water phase decreases. Otherwise, the saturation of the water phase increases, and the water channels become larger. On the meniscus in the water channel, the aqueous phase acts as a low-viscosity fluid to lubricate the oil phase flow, enabling the oil to separate from the inner walls and reach the start condition. Therefore, in addition to the driving force on the cross section, the viscous force of the water phase channel against the oil-water interface also promotes oil migration. In a more irregular pore section, the trapped oil can more easily migrate. Therefore, it is reasonable to believe that residual oil is easier to recover in porous media that have irregular pore sections.

3.3. Influence of the Cross Section on Non-Darcy Flow. The relationship between the pressure drop and the velocity of oil in capillary tubes of different cross sections is investigated to identify the influence of the microchannel cross section on the non-Darcy flow of the discontinuous oil phase. Figure 8 shows the relationship between the pressure drop and velocity after the trapped oil reached a steady state in the three capillaries. As a comparison, circular capillaries $(0.5 \mathrm{~mm}$ radius) are also included in the investigation. The lengths and properties of the trapped oil in these four cases refer to Section 2.2

Figure 8 shows the pressure-velocity curves of oil in circular, pentagonal, quadrilateral, and triangular tubes under steady-state conditions. In the circular capillary, the trapped oil completely separates from the inner wall and is enclosed by the water film when the flow in the tube enters the steady flow stage [3]. In this state, the oil moves forward in a capsule form. Under this condition, the pressure drop in the tube is linearly related to the velocity, as shown in the circular dotted line in Figure 8, and this trend indicates a Darcy flow. The curves of the other noncircular capillaries are nonlinear, indicating that the flow within them is a non-Darcy flow. At the same velocity, the pentagon capillary shows the largest pressure drop, while the triangle capillary has the smallest pressure drop. This phenomenon demonstrates that the trapped oil in triangular tubes flows more easily, regardless of whether the oil is in a start condition. This phenomenon is because, when there is a more irregular section shape, the saturation of water on the cross section increases, and the lubrication effect of the water on the oil is more obvious. Conversely, in the higher-order polygonal capillary section, the water saturation decreases and the resistance of the oil increases. When the polygon becomes circular, the resistance reaches a maximum. The three curves can be divided into two stages: as the velocity increases from zero, the pressure drop increases gradually and the slope decreases; and when a critical velocity is reached, the curve trend becomes linear. Based on the previous analysis, the two stages are considered to be nonstart and start conditions, respectively. At a less than critical velocity, the trapped oil remains in contact with the inner wall when it finally reaches a steady state. Meanwhile, among the three noncircular cases, the critical velocity of the most irregular cross section (triangular) is the minimum. As previously described, in porous media with more irregular pores, the residential oil will more easily migrate even when other process parameters remain the same.

\section{Conclusion}

In this research, a numerical study is carried out to elucidate the mechanism of the initiation and migration of trapped oil in capillaries with noncircular cross sections. Three typical 
cross sections are used to study the start process of trapped oil migration and the mechanism of the non-Darcy flow in irregular microchannels. This paper mainly found the following conclusions:

(1) In a noncircular cross-sectional capillary, the trapped oil maintains contact with the inner walls and remains in a nonstart condition under low velocity; with the increase of velocity, the area of the oil droplets that are encapsulated by water film increases and the amount of contact between the oil phase and the inner wall decreases. When the critical velocity is reached, the oil separates from the walls and moves forward as a capsule shape, while the oil-water interface is continuous

(2) The water phase in a noncircular capillary drives the trapped oil through two kinds of actions: the driving force on the cross section of the oil drop and the viscous force on the oil-water interfaces in the corners. When the cross section becomes a higher-order polygon, i.e., the cross section becomes more circular, the decreasing water channels in the corners result in their decreasing viscous force, thus affecting the initiation and migration of the trapped oil

(3) When the displacement velocity is less than the critical velocity, the oil stabilizes in a nonstart condition. At different displacement velocities, there are changes in the proportion of the oil that is wrapped by the water film and in the area directly in contact with the inner walls. At this stage, the relationship between the capillary pressure drop and the displacement velocity does not change linearly, and the flow is described as a non-Darcy flow

\section{Abbreviations}

MS-P: $\quad$ Mayer, Stowe, and Princen

CFD: $\quad$ Computational fluid dynamics

VOF method: Volume of fluid method.

\section{Data Availability}

The data used to support the findings of this study are available from the corresponding author upon request.

\section{Conflicts of Interest}

The authors declare that they have no conflicts of interest.

\section{Acknowledgments}

The authors gratefully acknowledge financial supports from the Natural Science Foundation of China (Nos. 51204198 and 51274225) and the National Basic Research Program of China (No. 2014CB239103).

\section{References}

[1] J. Li, Y. Liu, Y. Gao, B. Cheng, F. Meng, and H. Xu, "Effects of microscopic pore structure heterogeneity on the distribution and morphology of remaining oil," Petroleum Exploration and Development, vol. 45, no. 6, pp. 11121122, 2018.

[2] X. Gu, C. Pu, N. Khan, F. Wu, F. Huang, and H. Xu, "The visual and quantitative study of remaining oil microoccurrence caused by spontaneous imbibition in extra-low permeability sandstone using computed tomography," Fuel, vol. 237, pp. 152-162, 2019.

[3] M. Dong, Q. Fan, and L. Dai, "An experimental study of mobilization and creeping flow of oil slugs in a water-filled capillary," Transport in Porous Media, vol. 80, no. 3, pp. 455-467, 2009.

[4] L. Long, Y. Li, H. Gong, L. Xu, Q. Sang, and M. Dong, "Investigation of pressure drop of trapped oil in capillaries with circular cross-sections," Industrial \& Engineering Chemistry Research, vol. 57, no. 41, pp. 13866-13875, 2018.

[5] T. Cubaud and C.-M. Ho, "Transport of bubbles in square microchannels," Physics of Fluids, vol. 16, no. 12, pp. 45754585, 2004.

[6] D. Qian and A. Lawal, "Numerical study on gas and liquid slugs for Taylor flow in a T-junction microchannel," Chemical Engineering Science, vol. 61, no. 23, pp. 7609-7625, 2006.

[7] M. J. Fuerstman, A. Lai, M. E. Thurlow, S. S. Shevkoplyas, H. A. Stone, and G. M. Whitesides, "The pressure drop along rectangular microchannels containing bubbles," Lab on a Chip, vol. 7, no. 11, pp. 1479-1489, 2007.

[8] Z. Yu, O. Hemminger, and L.-S. Fan, "Experiment and lattice Boltzmann simulation of two-phase gas-liquid flows in microchannels," Chemical Engineering Science, vol. 62, no. 24, pp. 7172-7183, 2007.

[9] D. M. Fries, F. Trachsel, and P. R. von Rohr, "Segmented gasliquid flow characterization in rectangular microchannels," International Journal of Multiphase Flow, vol. 34, no. 12, pp. 1108-1118, 2008.

[10] S. Li, R. Chen, H. Wang et al., "Numerical investigation of the moving liquid column coalescing with a droplet in triangular microchannels using CLSVOF method," Science Bulletin, vol. 60, no. 22, pp. 1911-1926, 2015.

[11] Q. Liao, S. Li, R. Chen et al., "Coalescence with droplets caused acceleration of the liquid movement in microchannels," Industrial \& Engineering Chemistry Research, vol. 54, no. 3, pp. 1161-1169, 2015.

[12] C. Rong, L. Shuzhe, W. Hong et al., "Dynamic behavior of the liquid flow coalescing with a droplet in hydrophobic microchannels," Journal of Nanoscience and Nanotechnology, vol. 15, no. 4, pp. 2923-2931, 2015.

[13] F. Fairbrother and A. E. Stubbs, "Studies in electroendosmosis. Part VI. The "bubble-tube" method of measurement," Journal of the Chemical Society, pp. 527-529, 1935.

[14] R. N. Marchessault and S. G. Mason, "Flow of entrapped bubbles through a capillary," Industrial \& Engineering Chemistry, vol. 52, no. 1, pp. 79-84, 1960.

[15] F. P. Bretherton, "The motion of long bubbles in tubes," Journal of Fluid Mechanics, vol. 10, no. 2, pp. 166-188, 1961.

[16] G. I. Taylor, "Deposition of a viscous fluid on the wall of a tube," Journal of Fluid Mechanics, vol. 10, no. 2, p. 161, 1961. 
[17] J.-D. Chen, "Measuring the film thickness surrounding a bubble inside a capillary," Journal of Colloid and Interface Science, vol. 109, no. 2, pp. 341-349, 1986.

[18] T. Taha and Z. F. Cui, "Hydrodynamics of slug flow inside capillaries," Chemical Engineering Science, vol. 59, no. 6, pp. 11811190, 2004.

[19] T. Taha and Z. F. Cui, "CFD modelling of slug flow inside square capillaries," Chemical Engineering Science, vol. 61, no. 2, pp. 665-675, 2006.

[20] T. Taha and Z. F. Cui, "CFD modelling of slug flow in vertical tubes," Chemical Engineering Science, vol. 61, no. 2, pp. 676687, 2006.

[21] L. Dai and Y. Zhang, "Experimental study of oil-water twophase flow in a capillary model," Journal of Petroleum Science and Engineering, vol. 108, no. 4, pp. 96-106, 2013.

[22] L. Dai and X. Wang, "Numerical study on mobilization of oil slugs in capillary model with level set approach," Engineering Applications of Computational Fluid Mechanics, vol. 8, no. 3, pp. 422-434, 2014.

[23] L. Dai, G. Cheng, M. Dong, and Y. Zhang, "The influence of vibratory excitation on the oil slug mobilization in a capillary model," Journal of Petroleum Science Research, vol. 5, no. 1, pp. 1-13, 2016.

[24] S.-Y. Hsu and M. Hilpert, "Pore-scale visualization of the mobilization of a partially wetting droplet," Advances in Water Resources, vol. 95, pp. 235-245, 2016.

[25] W. B. Lindquist, S. M. Lee, D. A. Coker, K. W. Jones, and P. Spanne, "Medial axis analysis of void structure in threedimensional tomographic images of porous media," Journal of Geophysical Research: Solid Earth, vol. 101, no. B4, pp. 8297-8310, 1996.

[26] W. B. Lindquist, A. Venkatarangan, J. Dunsmuir, and T. F. Wong, "Pore and throat size distributions measured from synchrotron X-ray tomographic images of Fontainebleau sandstones," Journal of Geophysical Research: Solid Earth, vol. 105, no. B9, pp. 21509-21527, 2000.

[27] W. B. Lindquist, “The geometry of primary drainage," Journal of Colloid and Interface Science, vol. 296, no. 2, pp. 655-668, 2006.

[28] Y. Sholokhova, D. Kim, and W. Brent Lindquist, "Network flow modeling via lattice-Boltzmann based channel conductance," Advances in Water Resources, vol. 32, no. 2, pp. 205212, 2009.

[29] D. Wildenschild and A. P. Sheppard, "X-ray imaging and analysis techniques for quantifying pore-scale structure and processes in subsurface porous medium systems," Advances in Water Resources, vol. 51, pp. 217-246, 2013.

[30] P. Jia, M. Dong, and L. Dai, "Threshold pressure in arbitrary triangular tubes using RSG concept for all wetting conditions," Colloids and Surfaces A: Physicochemical and Engineering Aspects, vol. 302, no. 1-3, pp. 88-95, 2007.

[31] L. Long, Y. Li, and M. Dong, "Liquid-liquid flow in irregular triangular capillaries under different wettabilities and various viscosity ratios," Transport in Porous Media, vol. 115, no. 1, pp. 79-100, 2016.
[32] H. Joshi and L. Dai, "A numerical investigation on a flow of a viscous oil drop in water inside a small diameter capillary tube," in ASME 2012 International Mechanical Engineering Congress and Exposition, pp. 1047-1053, Houston, TX, USA, 2012.

[33] L. Long and B. Zhang, "The distribution of fluids in irregular capillary tubes: a new capillary model based on the single-corner capillary," Journal of Petroleum Exploration and Production Technology, vol. 8, no. 2, pp. 341-350, 2018. 

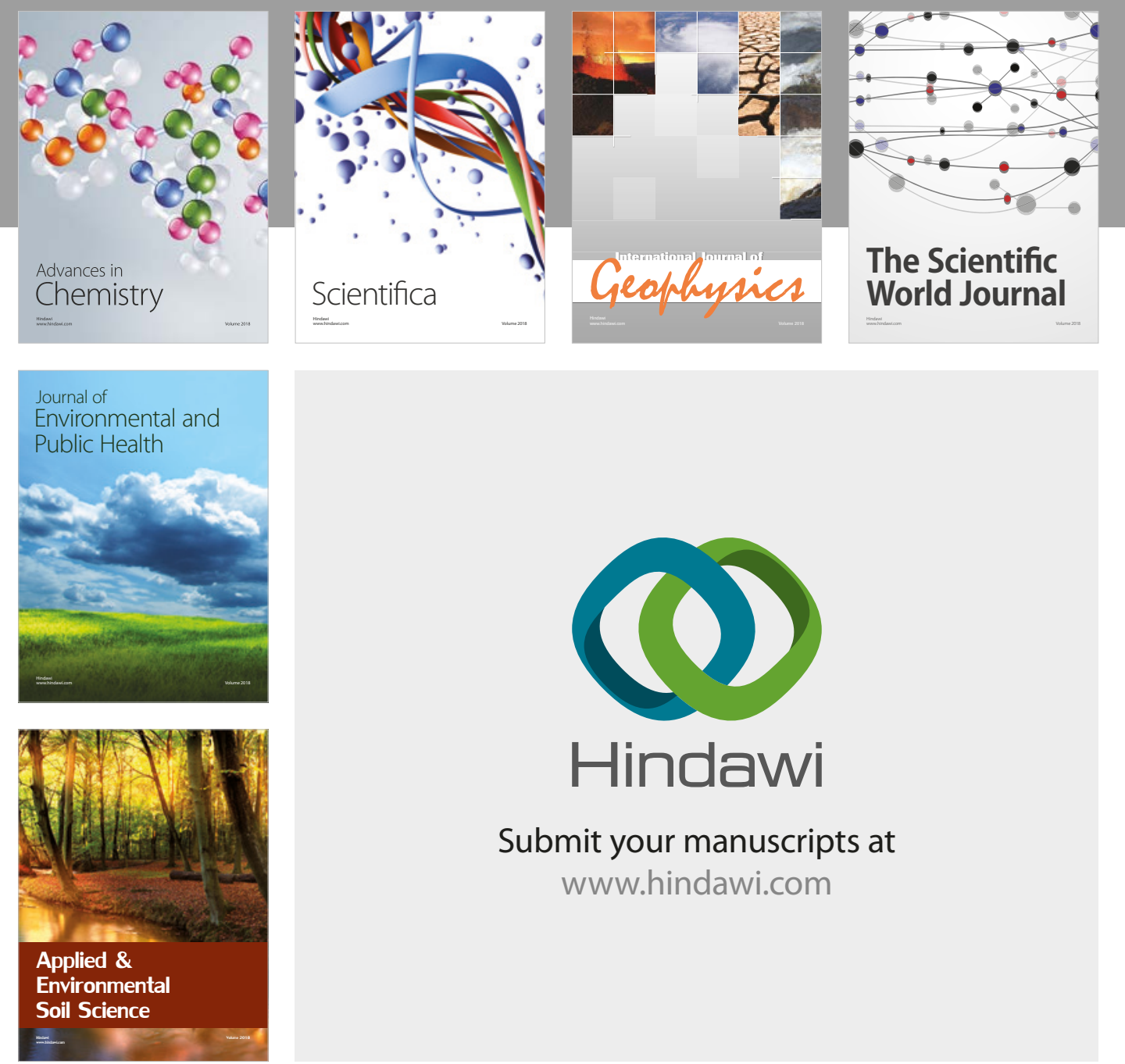

The Scientific

\section{World Journal}
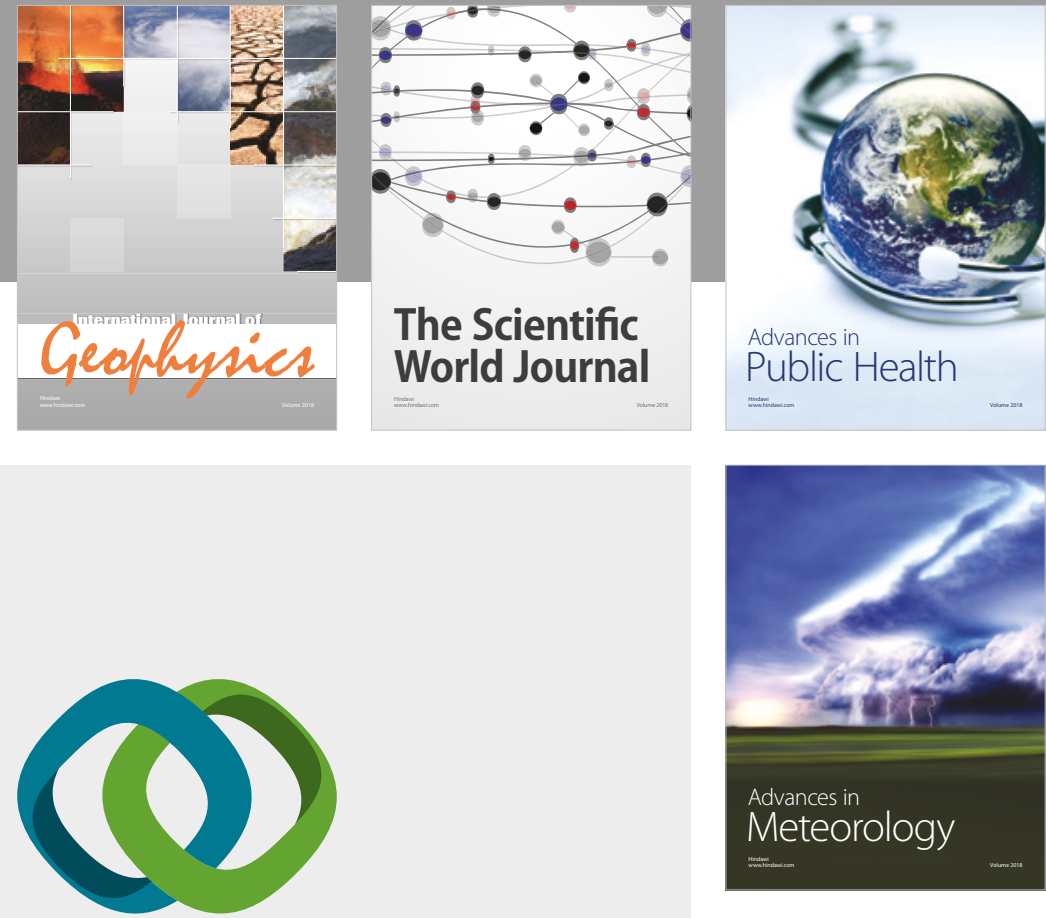

Advan

Public Health

\section{Hindawi}

Submit your manuscripts at

www.hindawi.com
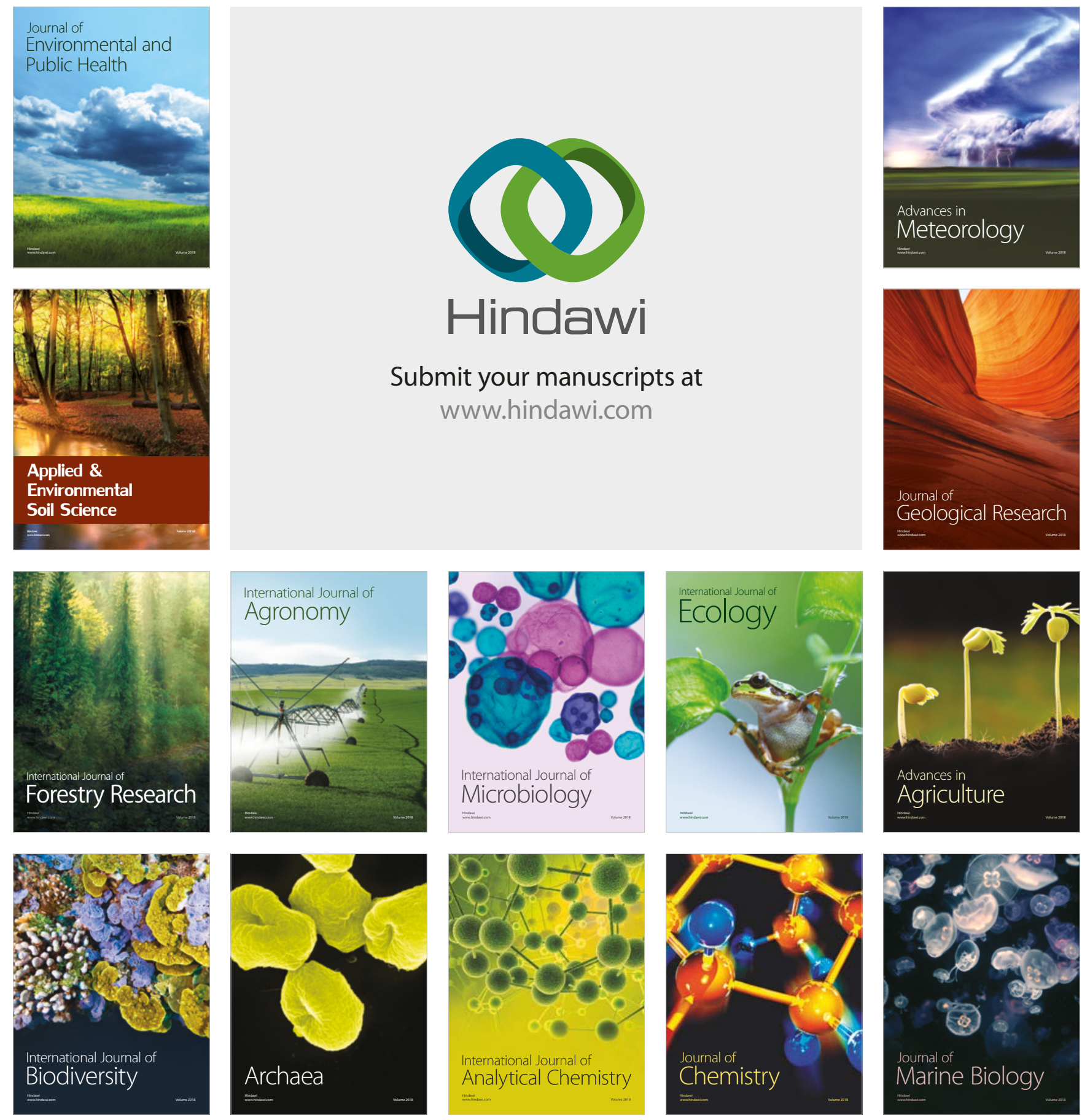